\title{
Factores Determinantes de la Sostenibilidad de las Agroempresas Asociativas Rurales ${ }^{1}$
}

\author{
Holmes Rodríguez Espinosa², Carlos Julián Ramírez Gómez ${ }^{3}$ y \\ Luis Fernando Restrepo Betancur ${ }^{4}$
}

\begin{abstract}
Resumen: este estudio tuvo como objetivo proponer un enfoque multidimensional de análisis de la sostenibilidad en la agroempresa asociativa rural. Se utilizó un muestreo dirigido seleccionando cuatro programas de acompañamiento al fortalecimiento de la asociatividad rural llevados a cabo en cuatro departamentos de Colombia: Antioquia, Cauca, Nariño y Valle del Cauca. Los datos fueron recolectados mediante 79 entrevistas semiestructuradas con diversos actores y posteriormente analizados utilizando análisis factorial. El estudio permitió construir un enfoque para el análisis de la sostenibilidad de organizaciones agroempresariales rurales a partir de la identificación de 50 variables que fueron agrupadas en dos componentes y tres subcomponentes: Socio organizacional (Capital social, Planeación organizacional e Integración territorial) y Empresarial (Económico, Productivo tecnológico y orientación al mercado), para la definición de un índice de sostenibilidad de la agroempresa rural (Isoar). El cálculo del Isoar permitió identificar que los dos subcomponentes menos destacados en todos los casos estudiados fueron el Productivo-Tecnológico y la Orientación al mercado, mientras en los casos más exitosos los componentes más destacados fueron el capital social y la integración territorial. Se concluye que el enfoque de sostenibilidad de las agroempresas asociativas rurales soportado en un modelo multidimensional constituye pilares para la formulación de políticas públicas.
\end{abstract}

Palabras-clave: agronegocios, asociaciones, capital social.

Abstract: this research aimed to propose a multidimensional approach to analyze associative agribusiness sustainability. Sampling was used selecting four support programs to strengthening rural associativity, carried out in four states in Colombia: Antioquia, Cauca, Nariño and Valle del Cauca. Data was collected through 79 semistructured interviews with various actors and subsequently analyzed using factor analysis. The study allowed to build up an approach to the associative agribusiness sustainability analysis from identifying 50 variables that were grouped in two components and three

1. Data de submissão: 29 de agosto de 2016. Data de aceite: 20 de agosto de 2017.

2. Universidad de Antioquia UdeA, Medellín, Antioquia, Colombia. E-mail: holmesrodriguez@gmail.com

3. Universidad de Antioquia UdeA, Medellín, Antioquia, Colombia. E-mail: cjulianmdr@gmail.com

4. Universidad de Antioquia UdeA, Medellín, Antioquia, Colombia. E-mail: frbstatistical@gmail.com 
sub-components: Socio-organizational (Social capital, Organizational planning and Territorial Integration) and Enterprise (Economic, Productive-technological and market orientation), to define an associative rural agribusiness sustainability index (Isoar). The Isoar calculation allows to identify productive-technological and market orientation sub-components as the less prominent in the studied cases, while social capital and territorial integration were the most prominent in the successful cases. It is concluded that the associative agribusiness sustainability approach, supported in a multidimensional model, is a contribution to public policy.

Key-words: agribusiness, associations, social capital, sustainability.

Clasificación JEL: L31.

DOI: http://dx.doi.org/10.1590/1234-56781806-94790560107

\section{Introducción}

Ante el incremento de la globalización, el sector agropecuario de América Latina viene afrontando amplios retos que implican nuevos marcos de política pública y un mejoramiento de la competitividad, que permitan aumentar la productividad, la reducción de la pobreza y la sostenibilidad del sector (SPIELMAN y KELEMEWORK, 2009). Esta circunstancia pone en evidencia la necesidad de promover acciones tendientes a la modernización del sector, por ejemplo a partir del desarrollo de agroempresas rurales, permitiendo movilizar las capacidades de los territorios, en las cuales están insertas (OECD, 2014), teniendo en cuenta por ejemplo, la importancia que este tipo de organizaciones tiene en la generación de empleo y aporte al PIB (MELGAREJO, VERA-COLINA y MORA-RIAPIRA, 2013).

Particularmente en Colombia, se ha buscado la promoción y el fortalecimiento de las empresas asociativas rurales, a través de diversas estrategias implementadas en los últimos 40 años; no obstante, aún persisten problemas de baja organización de los productores (SAC, 2010), y de un modelo de desarrollo caracterizado por el individualismo y la competencia de los agentes del mercado (RUEDA y MUÑOZ, 2011). En ese sentido las políticas públicas enfrentan diversos retos, en los cuales el sector académico debe tener un papel más protagónico, en particular en la profundización de marcos de análisis comprensivos, que permitan evaluar la perspectiva agroempresarial asociativa bajo enfoques más integrales, generando insumos para el mejoramiento de la política.
En tal sentido, es necesario repensar los modelos asociativos agroempresariales rurales, hacia una visión de integración multidimensional e incluyente, superando aquellos enfoques con mayores objetivos técnicos (producción, tecnología, infraestructura, mercados), permitiendo escalar a objetivos más de carácter social y colectivo (MURCIA, 2011), bajo una visión de abajo hacia arriba desde lo local territorial (RODRÍGUEZ, RAMÍREZy RESTREPO, 2015), en la cual el entorno social y cultural, aportan considerablemente a la permanencia de los sistemas empresariales en el tiempo (GARZÓN, AMAYA y CASTELLANOS, 2004). En este contexto, se plantea el concepto de sostenibilidad como el grado de fortalecimiento que alcanza una organización para subsistir de manera autónoma, una vez finaliza un proceso de acompañamiento institucional (RODRÍGUEZ y RAMÍREZ, 2016).

En consecuencia esta investigación tiene como objetivo identificar los factores determinantes de la sostenibilidad de la agroempresa asociativa rural, por medio de estudio de casos de programas de acompañamiento al fortalecimiento de la asociatividad llevados a cabo en varios departamentos de Colombia, que permita proponer un marco de análisis a partir de la construcción de un índice de sostenibilidad de las agroempresas asociativas rurales, con enfoque multidimensional.

\section{Fundamento teórico}

La asociatividad se puede definir como un proceso de trabajo colectivo en busca de objetivos comunes 
(BOLAÑOS, 1999) definidos de manera conjunta, a partir del desarrollo de principios y valores como confianza, compromiso, participación, liderazgo y comunicación (AMÉZAGA et al., 2013) para la obtención de resultados que de forma individual son más difíciles de alcanzar en aspectos productivos, organizativos y comerciales, haciendo un uso más eficiente de los recursos naturales, humanos, sociales, físicos y financieros disponibles (GOTTRET, JUNKIN y UGARTE, 2011).

La asociación agroempresarial como un modelo que busca impulsar la actividad económica o de servicios en beneficio de sus asociados, está compuesta fundamentalmente por dos aspectos: el primero el socio-organizativo sobre la base de aspectos con cimientos en el capital social; y el segundo el empresarial, con sus asuntos económicos, productivos y soportados en la planificación de plan operativo, estratégico y de agronegocios (AMÉZAGA et al., 2013). Adicionalmente para la actividad de las agroempresas asociativas rurales, se destacan las relaciones interorganizacionales e integración, a partir de vínculos que implican la acción integral, coordinada y consensuada, donde los agentes del territorio se convierten en socios para el desarrollo, mejorando la capacidad de las organizaciones y la competitividad territorial (SOLARTE, 2011).

En este sentido, el fortalecimiento de esquemas asociativos debe tener en cuenta además de los aspectos empresariales, el mejoramiento socio-organizativo (CENTRO PARA EL DESARROLLO DE CAPITAL HUMANO, 2007), en aspectos como las relaciones interpersonales, el análisis de estructuras sociales, capital social y cohesión de la población beneficiaria (EFENDIEV y SOROKIN, 2013). Una organización puede tener un plan de negocios muy bueno, pero, si carece de cohesión, la iniciativa difícilmente va a prosperar; de igual manera, una organización puede haber consolidado la confianza y el compromiso de los asociados, pero, si la gestión económica es deficiente, el negocio está destinado al fracaso (AMÉZAGA et al., 2013).

De hecho el mejoramiento de estas capacidades bien pueden representar ventajas de la asociatividad en aspectos económicos, al mejorar la capacidad de enfrentar las amenazas del entorno y contribuir al desarrollo socioeconómico por medio dela construcción de capital social y fortalecimiento del capital humano
(AMÉZAGA et al., 2013). Adicionalmente, las agroempresas asociativas rurales, vistas como un actor integrado territorialmente, permiten a los productores asociados beneficiarse de las economías de escala generadas de la acción colectiva, facilitando el acceso a asesoría técnica para la producción, acceso a materias primas más económicas y mayor facilidad en el acceso a servicios financieros (ÁLVAREZ et al., 2012).

Sin embargo los casos de éxito o fracaso frente al fomento de este tipo de organizaciones puede ser realmente variable en los territorios rurales. De hecho, la literatura científica ha reportado la existencia de diversos tipos de limitaciones que inciden en el desarrollo asociativo, tales como la dificultad para el trabajo en equipo y la deficiencia en la distribución de funciones y en la toma colectiva de decisiones (SZMULEWICZ, GUTIÉRREZ y WINKLER, 2012) al igual que la baja participación de los productores en los procesos organizativos (RIELLA y VITELLI, 2009); aspectos relacionados con la falta de planes estratégicos de las organizaciones, deficiencias en las capacidades de gestión administrativa y contable y debilidades en la capacitación de la base social (OSTERTAG, 2002).

Recientemente autores como Lemus-Aguilar y Hidalgo (2016) han propuesto la noción de sostenibilidad de las organizaciones como un marco de referencia a la capacidad desarrollada por la organización para innovar y permanecer en el tiempo, manteniéndose en el mercado con criterios de responsabilidad económica, social y ambiental.

El interés por abordar las problemáticas entorno a la cuestión agroempresarial ruraly su relación con variables que pueden incidir y explicar su comportamiento, entre otras razones, pone en evidencia algunos estudios y metodologías de investigación empleadas para contribuir a comprender determinantes que inciden en el sostenimiento de estas organizaciones.

Por ejemplo en el trabajo de Amoah-Mensah (2013) se abordó la perspectiva del desempeño de pequeñas y medianas empresas rurales desde la teoría basada en recursos discutiendo la existencia de recursos internos tanto tangibles como intangibles así como recursos externos, sobre los cuales mediante la identificación de diversas variables y modelo de regresión lineal múltiple se constituyeron indicadores de evaluación, destacando la experiencia acumulada de las personas como uno de los recursos estratégicos más importantes. 
En la misma visión del análisis sobre relaciones de causalidad entre variables se destaca el trabajo de Quang y Itagaki (2012), quienes también mediante modelo econométrico explicaron como aspectos relacionados con las dotaciones de infraestructura, cercanía, tecnología, reglas y regulaciones, tenencia de la tierra, disponibilidad de capital, servicios públicos, el rol de proveedores locales y externos, así como un asunto de capacidades, entre otros factores contribuyen al desempeño económico de agro emprendimientos rurales.

El análisis de causalidad en el desempeño agroempresarial rural es abordado también por Bialoskorski (2007), quien al plantear el estudio de empresas cooperativas agropecuarias establece que el costo de oportunidad de la participación de sus socios frente al incentivo económico de la participación social tiene influencia en el desempeño económico de estas empresas presentando una relación inversamente proporcional corroborado con su modelo de regresión.

Por su parte autores como Ensslin, Ensslin, Imlau y Chaves (2014), realizaron un análisis bibliométrico de tendencias sobre evaluación del desempeño de empresas cooperativas agropecuarias, concluyendo que es aún un campo poco explorado en la literatura científica, destacando que para una mejor gestión y garantizar el éxito de las actividades de las empresas cooperativas, se requiere de controles, técnicas y métodos de evaluación de desempeño como cualquier otra entidad.

Dado entonces el interés por evaluar las agroempresas rurales, en Colombia se aplicó el método de medición del desempeño de organizaciones a partir del Índice de Capacidad Organizacional ICO (MINISTERIO DE AGRICULTURA Y DESARROLLO RURAL, 2003), por medio del análisis de 43 indicadores en 15 categorías y 5 ejes con una ponderación preestablecida y su cálculo en escala de 0 a 100; y la Valoración del Estado Organizacional VEO (USAID, $\mathrm{n} / \mathrm{d}$.), a partir del análisis de 63 indicadores en 21 categorías y 5 ejes con esquemas de ponderación.

Sin embargo poder entender el fenómeno de la permanencia de organizaciones empresariales rurales, una noción más integral implica una visión tanto de la ciencia de la estrategia empresarial y organizacional, así como la teoría de la acción colectiva (MUÑOZ, SANTOYO y FLORES, 2010). En consecuencia los autores analizaronla correlación entreelgradoadopción de la propuesta de siete principios cooperativos en 75 organizaciones con relación a la mezcla de servicios, abarcando variables de la dimensión organizacional e institucional (principios, valores, propuesta de valor), y variables de la acción colectiva (redes, instituciones, variables colectivas, confianza y reciprocidad).

Aunque se evidencia en la literatura una tendencia entorno al interés por el análisis del desempeño o permanencia de las agroempresas rurales, son pocos los estudios que abordan el asunto en la perspectiva de las organizaciones agroempresariales rurales generando allí amplias posibilidades de profundización. Del mismo modo poder interpretar de manera más multidimensional algunos factores que puedan explicar por qué algunas organizaciones agroempresariales rurales de carácter asociativo más allá de su desempeño, logran permanecer en el tiempo sosteniéndose en sus entornos y territorios específicos, aún constituye brechas en el conocimiento que deben ser ampliadas ya que pueden constituir insumos para el fomento de políticas públicas.

\section{Metodología}

\subsection{Enfoque metodológico y selección de casos de estudio}

Se realizó una investigación con enfoque metodológico cualitativo, de carácter interpretativo (DENZIN y LINCOLN, 1994; LAMNEK, 2005; NOELLE-NEUMANN y PETERSEN, 2005), utilizando las técnicas de estudio de casos, encuesta y análisis de archivos (YIN, 2013), por medio de las cuales se realizó una evaluación completa de los acontecimientos en un marco geográfico definido en un período determinado, buscando una lógica emergente que vincule e integre las respuestas obtenidas con el marco conceptual establecido.

Se utilizó un muestreo dirigido con base en criterios definidos a fin de cubrir un amplio rango de situaciones presentes en la zona de estudio (BORG, 1989), lo cual resulta más apropiado para el entendimiento de la situación estudiada, que utilizar muestras representativas (ARBER, 1993). Se definieron como criterios para la selección de la muestra, los siguientes: integrada por pequeños productores, participación en programas de apoyo al fortalecimiento asociativo, permanencia en el tiempo y disponibilidad de información sobre la organización. 
A partir del cumplimiento de estos criterios se seleccionaron 4 programas de acompañamiento al fortalecimiento de la asociatividad llevados a cabo en los departamentos (División político-administrativa equivalente a Estados) de: Antioquia, Cauca, Nariño y Valle del Cauca, por cuanto ofrecían las mejores oportunidades para aprender y se contaba con la facilidad de acceso (MARRADI, ARCHENTI y PIOVANI, 2007). La estrategia seleccionada para realizar el proceso investigativo fue el estudio de casos múltiples, utilizando un diseño de múltiples casos y múltiples unidades de análisis (YIN, 2013).

\subsection{Instrumentos de recolección de información}

Los instrumentos para la recolección de información fueron las entrevistas en profundidad y la observación directa en donde se construyó un diario de campo permitiendo sistematizar diversos aspectos observados. Para la recolección de los datos también se llevaron a cabo entrevistas semi-estructuras con personal de apoyo de las instituciones acompañantes (9) y personal administrativo (54) y productores de las organizaciones (16) estudiadas y consulta de información documental de las organizaciones.

Las entrevistas semiestructuradas se construyeron a partir de la propuesta de análisis multidimensional de las organizaciones agroempresariales rurales, para lo cual se determinaron 50 variables provenientes de la revisión total de literatura. Estas variables fueron categorizadas en dos componentes, cada uno con tres subcomponente: Socio organizacional (Capital social,
Planeación organizacional e Integración territorial) y Empresarial (Económico, Productivo tecnológico y orientación al mercado).

\subsection{Análisis de la información}

Posteriormente para la elaboración del índice se utilizó el análisis factorial, con base en la metodología para el cálculo de otros índices reportados en la literatura (AYALA, ITURRALDE y RODRÍGUEZ, 2000; MARTÍNEZ-PELLITERO y BAUMERT, 2003). Para ello se tuvo en cuenta las comunalidades, definidas como la proporción de la varianza explicada por los factores comunes asociados con la variable, las cuales constituyeron las ponderaciones empleadas en el constructor del índice. Posteriormente se llevó a cabo el cálculo del Índice de sostenibilidad de la organización agroempresarial rural (Isoar) para las organizaciones estudiadas, realizando un análisis de conglomerados. Se emplearon los siguientes paquetes estadísticos: SAS University Virtual App y Factor 8.

\section{Resultados}

\subsection{Características, evolución y desarrollo de los casos estudiados}

Las experiencias estudiadas presentaron diferencias importantes en cuanto al contexto socioeconómico y cultural y la fundamentación de la experiencia en relación con su enfoque y el tipo de financiación (Tabla 1), sin embargo por su importancia en cada

Tabla 1. Características de los procesos de fortalecimiento asociativo estudiados

\begin{tabular}{|c|c|c|c|c|c|}
\hline Región & Cant & Contexto socioeconómico & Características & Subsector & $\begin{array}{l}\text { Recursos } \\
\text { de apoyo }\end{array}$ \\
\hline Nariño & 12 & $\begin{array}{l}\text { Población de economía campesi- } \\
\text { na, con experiencia en la produc- } \\
\text { ción de cebada }\end{array}$ & $\begin{array}{l}\text { Programa de reconversión productiva. In- } \\
\text { tervención en: fortalecimiento social, gene- } \\
\text { ración de ingresos, asistencia técnica, acceso } \\
\text { al mercado y gestión administrativa }\end{array}$ & Hortalizas & Privados \\
\hline Cauca & 14 & $\begin{array}{l}\text { Población de economía campesi- } \\
\text { na, localizados en zonas afectadas } \\
\text { por el conflicto armado }\end{array}$ & $\begin{array}{l}\text { Programa de desarrollo rural integrado. In- } \\
\text { tervención en: generación de ingresos, asis- } \\
\text { tencia técnica y acceso al mercado }\end{array}$ & $\begin{array}{c}\text { Frutas y } \\
\text { Hortalizas }\end{array}$ & Privados \\
\hline $\begin{array}{l}\text { Valle del } \\
\text { Cauca }\end{array}$ & 18 & $\begin{array}{l}\text { Población de economía campe- } \\
\text { sina, con experiencia en cultivo } \\
\text { permanentes }\end{array}$ & $\begin{array}{l}\text { Programa de desarrollo rural integrado. In- } \\
\text { tervención en: acceso al mercado y gestión } \\
\text { administrativa }\end{array}$ & Frutas & Públicos \\
\hline Antioquia & 10 & $\begin{array}{l}\text { Población de economía campesi- } \\
\text { na, localizados en zonas afectadas } \\
\text { por el conflicto armado }\end{array}$ & $\begin{array}{l}\text { Programas de alianzas para mejorar las con- } \\
\text { diciones de acceso al mercado. Intervención } \\
\text { en: asistencia técnica y acceso al mercado }\end{array}$ & $\begin{array}{l}\text { Frutas, } \\
\text { hortalizas, } \\
\text { café, leche }\end{array}$ & Públicos \\
\hline
\end{tabular}

Fuente: Elaboración propia (2015). 
departamento, se convierten en un buen referente para el estudio de la sostenibilidad de la organización agroempresarial rural.

\subsection{Enfoque de análisis de la sostenibilidad de organizaciones agroempresariales rurales}

Para el estudio de la sostenibilidad de las organizaciones agroempresariales rurales, se construyó un modelo multidimensional (Figura 1), en el cual se plantea la sostenibilidad soportada en cuatro pilares: capital social, planeación organizacional, económico, productivo-tecnológico; relacionados con dos aspectos del entorno: integración territorial y orientación al mercado (RUGELES y JOLLY, 2006).

Teniendo en cuenta la diversidad en los tipos de intervención llevados a cabo en cada departamento y las diferencias socioeconómicas y culturales existentes entre ellos, los resultados en términos del avance logrado en los factores relacionados con la sostenibilidad de las organizaciones agroempresariales, fueron diferentes (Tabla 2).

Para determinar el peso de cada subcomponente en la explicación de la sostenibilidad de la organizaciones agroempresariales rurales, se realizó el análisis del factor por medio de la técnica de componentes principales y posteriormente el análisis de comunalidad por subcomponente (Tabla 3).

Finalmente se realizó el calculó para cada subcomponente ponderando el factor por la comunalidad (Tabla 4).

Con base en estos resultados se definió la fórmula para calcular el Isoar (Índice de sostenibilidad de las organizaciones agroempresariales rurales):

$$
\begin{aligned}
& \text { ISOAR }=0,140 \mathrm{CS}+0,185 \mathrm{PO}+0,172 \mathrm{IT}+ \\
& +0,165 \mathrm{E}+0,175 \mathrm{PT}+0,163 \mathrm{OM}
\end{aligned}
$$

En el cual: CS: Capital Social; PO: Planeación organizacional; IT: Integración territorial; E: Económico; PT: Productivo-Tecnológico; OM: Orientación al mercado.

Figura 1. Modelo multidimensional para el análisis de la consolidación de la asociatividad agroempresarial rural

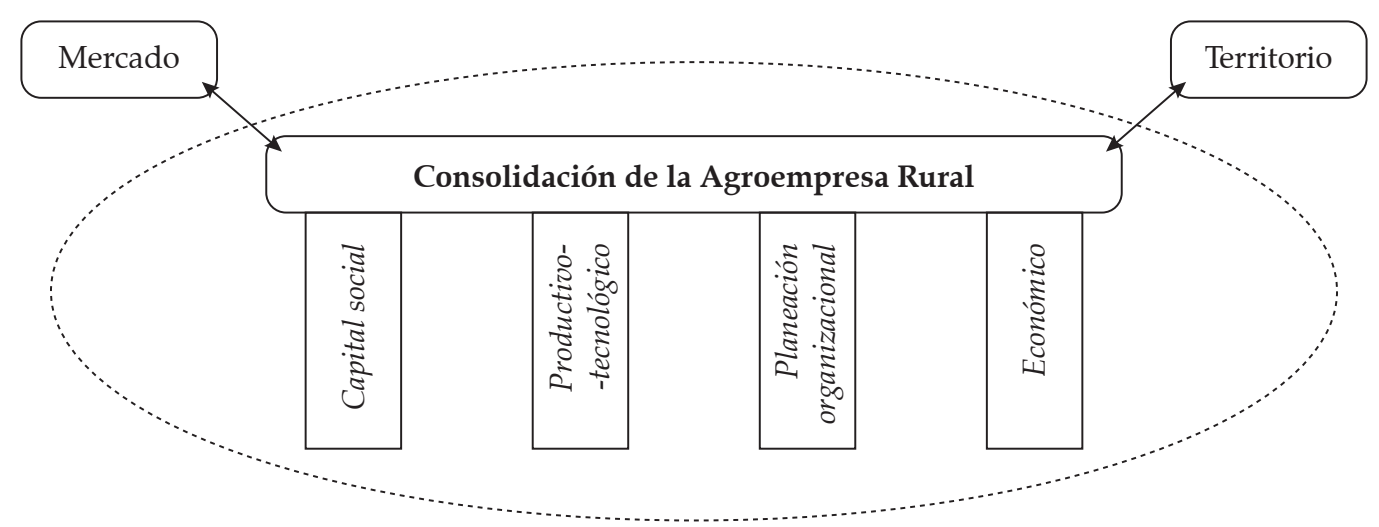

Fuente: Elaboración propia (2017).

\begin{tabular}{|c|c|c|c|c|c|c|}
\hline & & Aspecto & A & $\mathrm{C}$ & $\mathbf{N}$ & V \\
\hline \multirow{7}{*}{ 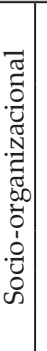 } & \multirow{7}{*}{ 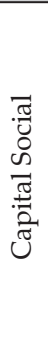 } & Estrategias para la generación de relaciones de confianza & $\mathrm{X}$ & $\mathrm{X}$ & $\mathrm{X}$ & \\
\hline & & Uso de metodologías participativas para involucrar a los productores en la identificación de necesidades & & $\mathrm{X}$ & $\mathrm{X}$ & \\
\hline & & $\begin{array}{l}\text { Uso de metodologías participativas para involucrar a los productores en el planteamiento de alter- } \\
\text { nativas de solución a sus necesidades }\end{array}$ & & $\mathrm{X}$ & & \\
\hline & & $\begin{array}{l}\text { Existencia de reglas adecuadas al interior de la organización para evitar acciones oportunistas por } \\
\text { parte de los asociados }\end{array}$ & $\mathrm{X}$ & $\mathrm{X}$ & $\mathrm{X}$ & $\mathrm{X}$ \\
\hline & & Mecanismos de intercambio de conocimientos y experiencias entre los asociados & $\mathrm{X}$ & $\mathrm{X}$ & $\mathrm{X}$ & \\
\hline & & Participación activa de los productores en los espacios administrativos & $\mathrm{X}$ & $\mathrm{X}$ & & \\
\hline & & Participación activa de los productores en la toma de decisiones & $\mathrm{X}$ & $\mathrm{X}$ & & \\
\hline
\end{tabular}

Tabla 2. Buenas prácticas para el fortalecimiento de la asociatividad 


\begin{tabular}{|c|c|c|c|c|c|c|}
\hline & Aspecto & A & $\mathrm{C}$ & $\mathbf{N}$ & $\mathbf{V}$ \\
\hline \multirow{18}{*}{ 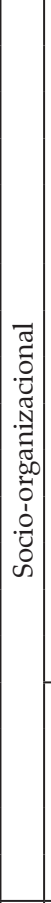 } & \multirow{14}{*}{ 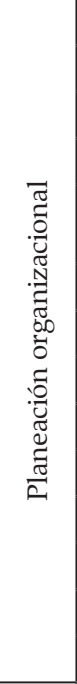 } & Proceso organizativo que surge por iniciativa de los beneficiarios & $\mathrm{X}$ & & & $\mathrm{X}$ \\
\hline & & Definición clara del objetivo de trabajo colectivo & $\mathrm{X}$ & $\mathrm{X}$ & $\mathrm{X}$ & $\mathrm{X}$ \\
\hline & & Nivel educativo medio de los integrantes de la organización & $\mathrm{X}$ & & & $x$ \\
\hline & & Existencia de una visión de futuro compartida & $x$ & $\mathrm{X}$ & $\mathrm{X}$ & \\
\hline & & $\begin{array}{l}\text { Estrategias de comunicación para mantener informados a los productores sobre el quehacer de la } \\
\text { organización }\end{array}$ & & & & \\
\hline & & Realización de reuniones periódicas para mantener informados a los productores & $\mathrm{X}$ & $x$ & $\mathrm{X}$ & \\
\hline & & Distribución de funciones entre los asociados por medio de comités y comisiones & & $X$ & & \\
\hline & & Elaboración del plan estratégico & $\mathrm{X}$ & $\mathrm{X}$ & $\mathrm{X}$ & $\mathrm{X}$ \\
\hline & & Elaboración del plan operativo & $\mathrm{X}$ & $X$ & $X$ & \\
\hline & & Prestación de servicios a los productores & $\mathrm{X}$ & $x$ & $\mathrm{X}$ & $\mathrm{X}$ \\
\hline & & Promoción del desarrollo de capacidades de liderazgo & $\mathrm{X}$ & $x$ & $\mathrm{X}$ & \\
\hline & & Promoción del desarrollo de capacidades para la acción colectiva & $\mathrm{X}$ & $\mathrm{X}$ & $\mathrm{X}$ & \\
\hline & & Procesos de capacitación & $\mathrm{X}$ & $\mathrm{X}$ & $x$ & $\mathrm{X}$ \\
\hline & & Tenencia de infraestructura para operación de la organización & $\mathrm{X}$ & $x$ & $\mathrm{X}$ & $\mathrm{X}$ \\
\hline & \multirow{4}{*}{ 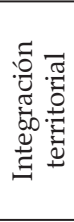 } & Pertenencia a redes de cooperación con otras organizaciones de productores & $\mathrm{X}$ & & $\mathrm{X}$ & $\mathrm{X}$ \\
\hline & & $\begin{array}{l}\begin{array}{l}\text { Integración hacia atrás de la cadena productiva para proveeduría de insumos a los sistemas productivos } \\
\text { (semillas, abonos, etc.) }\end{array} \\
\end{array}$ & $x$ & $\mathrm{X}$ & $X$ & \\
\hline & & Mecanismos de intercambio de conocimientos y experiencias con otras organizaciones & $\mathrm{X}$ & $X$ & $X$ & $\mathrm{X}$ \\
\hline & & Capacidad de gestión para el acceso a recursos de convocatorias públicas y privadas de proyectos & $\mathrm{X}$ & $x$ & $\mathrm{X}$ & $\mathrm{X}$ \\
\hline \multirow{25}{*}{ 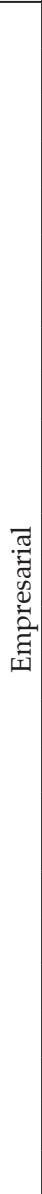 } & \multirow{10}{*}{ 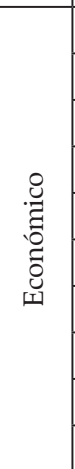 } & Elaboración del plan de negocios & $\mathrm{X}$ & $X$ & $\mathrm{X}$ & $\mathrm{X}$ \\
\hline & & Fondo rotatorio para apoyar a los productores en la compra de insumos & $\mathrm{X}$ & $\mathrm{X}$ & $\mathrm{X}$ & \\
\hline & & Mejoramiento de los ingresos de los productores por la optimización del canal de comercialización & $X$ & $X$ & $X$ & $\mathrm{X}$ \\
\hline & & Existencia de cuotas de sostenimiento & $\mathrm{X}$ & & $\mathrm{X}$ & $\mathrm{X}$ \\
\hline & & Existencia de comisión por venta por unidad de producto & $\mathrm{X}$ & $\mathrm{X}$ & $\mathrm{X}$ & $\mathrm{X}$ \\
\hline & & Proveeduría de insumos como mecanismo para la generación de ingresos & $\mathrm{X}$ & & $\mathrm{X}$ & \\
\hline & & Definición clara de la estructura de costos de la organización & $X$ & $X$ & $X$ & $\mathrm{X}$ \\
\hline & & Establecimiento claro del punto de equilibrio & $\mathrm{X}$ & $\mathrm{X}$ & $\mathrm{X}$ & $\mathrm{X}$ \\
\hline & & Definición de los márgenes de comercialización & $\mathrm{X}$ & $\mathrm{X}$ & $\mathrm{X}$ & $\mathrm{X}$ \\
\hline & & Seguimiento y control al cumplimiento de los márgenes de comercialización & $\mathrm{X}$ & & $\mathrm{X}$ & \\
\hline & \multirow{8}{*}{ 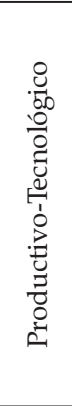 } & Asesoría en gestión empresarial orientada a la sostenibilidad & & $X$ & $X$ & $\mathrm{X}$ \\
\hline & & Prestación del servicio de asistencia técnica para el mejoramiento de los sistemas de producción & $\mathrm{X}$ & $\mathrm{X}$ & $\mathrm{X}$ & $\mathrm{X}$ \\
\hline & & Programas de promoción e implementación de buenas prácticas agropecuarias & $x$ & $X$ & $X$ & \\
\hline & & Prestación de servicios de asistencia técnica para diversificar los ingresos & & & $\mathrm{X}$ & \\
\hline & & Análisis de costos de producción & & & $X$ & \\
\hline & & Se cuenta con un análisis del impacto ambiental de las actividades realizadas por la organización & & & & \\
\hline & & Se cuenta con un plan de mitigación del impacto ambiental & & & & \\
\hline & & $\begin{array}{l}\text { Se ejecuta, se realiza seguimiento y se evalúa el cumplimiento del plan de mitigación del impacto } \\
\text { ambiental }\end{array}$ & & & & \\
\hline & \multirow{7}{*}{ 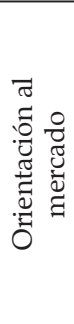 } & Comercialización del $100 \%$ de su producción a través de la asociación & $x$ & $X$ & $X$ & \\
\hline & & Planificación de la oferta de productos acordes con los requerimientos del mercado & & $\mathrm{x}$ & $\mathrm{X}$ & \\
\hline & & Existencia de diversidad de canales de comercialización & $\mathrm{X}$ & $X$ & $X$ & $\mathrm{X}$ \\
\hline & & Estrategias de diferenciación y agregación de valor a los productos & $\mathrm{X}$ & & $\mathrm{X}$ & $\mathrm{X}$ \\
\hline & & Portafolio diversificado de productos & & $X$ & $X$ & $X$ \\
\hline & & Estrategias para la gestión de calidad de los productos acordes con las exigencias del mercado & $\mathrm{X}$ & $\mathrm{X}$ & $\mathrm{X}$ & \\
\hline & & Capacidad financiera para pagar al productor en tiempo razonable & & & & \\
\hline
\end{tabular}

A: Antioquia; C: Cauca; N: Nariño; V: Valle del Cauca.

Fuente: Elaboración propia (2015). 
114 Factores Determinantes de la Sostenibilidad de las Agroempresas Asociativas Rurales

Tabla 3. Análisis del factor por tema

\begin{tabular}{|c|c|c|c|c|c|c|c|c|c|c|c|c|}
\hline \multirow[t]{2}{*}{ Variable } & \multicolumn{2}{|c|}{$\begin{array}{l}\text { Capital } \\
\text { Social }\end{array}$} & \multicolumn{2}{|c|}{$\begin{array}{c}\text { Proceso } \\
\text { Organizativo }\end{array}$} & \multicolumn{2}{|c|}{$\begin{array}{c}\text { Integración } \\
\text { Territorial }\end{array}$} & \multicolumn{2}{|c|}{ Económico } & \multicolumn{2}{|c|}{$\begin{array}{l}\text { Productivo- } \\
\text {-Técnico }\end{array}$} & \multicolumn{2}{|c|}{$\begin{array}{l}\text { Orientación al } \\
\text { Mercado }\end{array}$} \\
\hline & $F 1$ & $F 2$ & $F 1$ & $F 2$ & $F 1$ & $F 2$ & $F 1$ & $F 2$ & $F 1$ & $F 2$ & $F 1$ & $F 2$ \\
\hline 1 & 0.90 & & & 0.56 & & 0.93 & & & & & 0.84 & \\
\hline 2 & 0.73 & & 0.62 & & 0.87 & & 0.71 & & 0.81 & & 0.89 & \\
\hline 3 & 0.73 & & & 0.67 & 0.69 & & 0.81 & & 0.89 & & 0.81 & \\
\hline 4 & 0.84 & & 0.88 & & 0.88 & & & 0.78 & 0.73 & & & 0.93 \\
\hline 5 & 0.90 & & & 0.79 & & & 0.85 & & & 0.87 & 0.71 & \\
\hline 6 & & 0.60 & 0.88 & & & & & 0.80 & & & 0.78 & \\
\hline 7 & & 0.60 & & & & & & & & & & \\
\hline 8 & & & 0.70 & & & & & & & & & \\
\hline 9 & & & 0.66 & & & & 0.81 & & & & & \\
\hline 10 & & & 0.90 & & & & & & & & & \\
\hline 11 & & & 0.92 & & & & & & & & & \\
\hline 12 & & & 0.93 & & & & & & & & & \\
\hline 13 & & & & -0.58 & & & & & & & & \\
\hline 14 & & & 0.77 & & & & & & & & & \\
\hline$\%$ & 0.66 & 0.19 & 0.47 & 0.16 & 0.51 & 0.30 & 0.43 & 0.22 & 0.48 & 0.24 & 0.55 & 0.20 \\
\hline$A c u(\%)$ & \multicolumn{2}{|c|}{0.85} & \multicolumn{2}{|c|}{0.63} & \multicolumn{2}{|c|}{0.81} & \multicolumn{2}{|c|}{0.65} & \multicolumn{2}{|c|}{0.72} & \multicolumn{2}{|c|}{0.75} \\
\hline \multicolumn{13}{|c|}{ Comunalidades por tema } \\
\hline 1 & \multicolumn{2}{|c|}{0.936} & \multicolumn{2}{|c|}{0.781} & \multicolumn{2}{|c|}{0.884} & \multicolumn{2}{|c|}{0.608} & \multicolumn{2}{|c|}{0.496} & \multicolumn{2}{|c|}{0.753} \\
\hline 2 & \multicolumn{2}{|c|}{0.822} & \multicolumn{2}{|c|}{0.736} & \multicolumn{2}{|c|}{0.801} & \multicolumn{2}{|c|}{0.615} & \multicolumn{2}{|c|}{0.706} & \multicolumn{2}{|c|}{0.861} \\
\hline 3 & & & & & & & & & & & & \\
\hline 4 & & & & & & & & & & & & \\
\hline 5 & & & & & & & & & & & & \\
\hline 6 & & & & & & & & & & & & \\
\hline 7 & & & & & & & & & & & & \\
\hline 8 & & & & & & & & & & & & \\
\hline 9 & & & & & & & & & & & & \\
\hline 10 & & & & & & & & & & & & \\
\hline 11 & & & & & & & & & & & & \\
\hline 12 & & & & & & & & & & & & \\
\hline 13 & & & & & & & & & & & & \\
\hline 14 & & & & & & & & & & & & \\
\hline
\end{tabular}

Fuente: Elaboración propia (2015).

Tabla 4. Análisis del factor ponderando las comunalidades

\begin{tabular}{|c|c|c|c|c|c|}
\hline Sub-componente & Factor 1 & Factor 2 & Comunalidad & Global & Normalizado \\
\hline Capital Social & 0.846 & 0.001 & 0.716 & 6 & 0,140 \\
\hline Proceso Organizativo & 0.745 & -0.624 & 0.946 & 1 & 0,185 \\
\hline Integración Territorial & 0.521 & 0.780 & 0.881 & 3 & 0,172 \\
\hline Económico & 0.811 & -0.428 & 0.842 & 4 & 0,165 \\
\hline Productivo-Técnico & 0.856 & 0.399 & 0.893 & 2 & 0,175 \\
\hline Orientación al Mercado & 0.911 & 0.069 & 0.835 & 5 & 0,163 \\
\hline$\%$ Explicado & 0.6280 & 0.2246 & & & \\
\hline$\%$ Acumulado & \multicolumn{2}{|c|}{0.8526} & & & \\
\hline
\end{tabular}

Fuente: Elaboración propia (2015). 


\subsection{Análisis de la sostenibilidad de las} organizaciones agroempresariales estudiadas

Con base en la fórmula la propuesta, se calculó el Isoar de las organizaciones estudiadas (Tabla 5).

El análisis de conglomerados permitió identificar 3 grupos de organizaciones de acuerdo con el Isoar (Figura 2). El primer grupo de organizaciones se caracteriza por tener alto capital social, planeación organizacional, apoyo técnico productivo y orientación al mercado. El segundo clúster de organizaciones se caracterizó por tener alta participación organizacional y acceso al mercado. El tercer grupo lo constituyen aquellas organizaciones con bajo nivel de capital social, planeación organizacional, apoyo técnico productivo y orientación al mercado (Figura 3).

Tabla 5. ISOAR (Indice de sostenibilidad de las organizaciones agroempresariales rurales) por departamento y por sub-componente

\begin{tabular}{lcccccccc}
\hline \multicolumn{1}{c}{ Departamento } & ISOAR & CS & PO & IT & E & PT & OM \\
\hline Antioquia & $0,44 b^{*}$ & 0,46 & 0,55 & 0,80 & 0,48 & 0,14 & 0,19 \\
Cauca & $0,70 \mathrm{a}$ & 1,00 & 0,79 & 0,68 & 0,70 & 0,38 & 0,71 \\
Nariño & $0,75 \mathrm{a}$ & 0,57 & 0,73 & 1,00 & 0,94 & 0,55 & 0,69 \\
Valle del Cauca & $0,27 \mathrm{~b}$ & 0,10 & 0,34 & 0,38 & 0,44 & 0,14 & 0,18 \\
\hline Promedio & 0,52 & 0,50 & 0,58 & 0,67 & 0,63 & 0,29 & 0,43 \\
\hline Valor de p** & $<0,0001$ & $<0,0001$ & $<0,0001$ & $<0,0001$ & $<0,0001$ & $<0,0001$ & $<0,0001$ \\
\hline
\end{tabular}

*Letras distintas indican diferencia significativa con base en el análisis canónico derivado del MANOVA.

** Análisis multivariado de la varianza (MANOVA)Wilks' Lambda; Pillai's Trace; Hotelling-Lawley T; Roy's Greatest R.

Fuente: Elaboración propia (2015).

Figura 2. Análisis de conglomerados

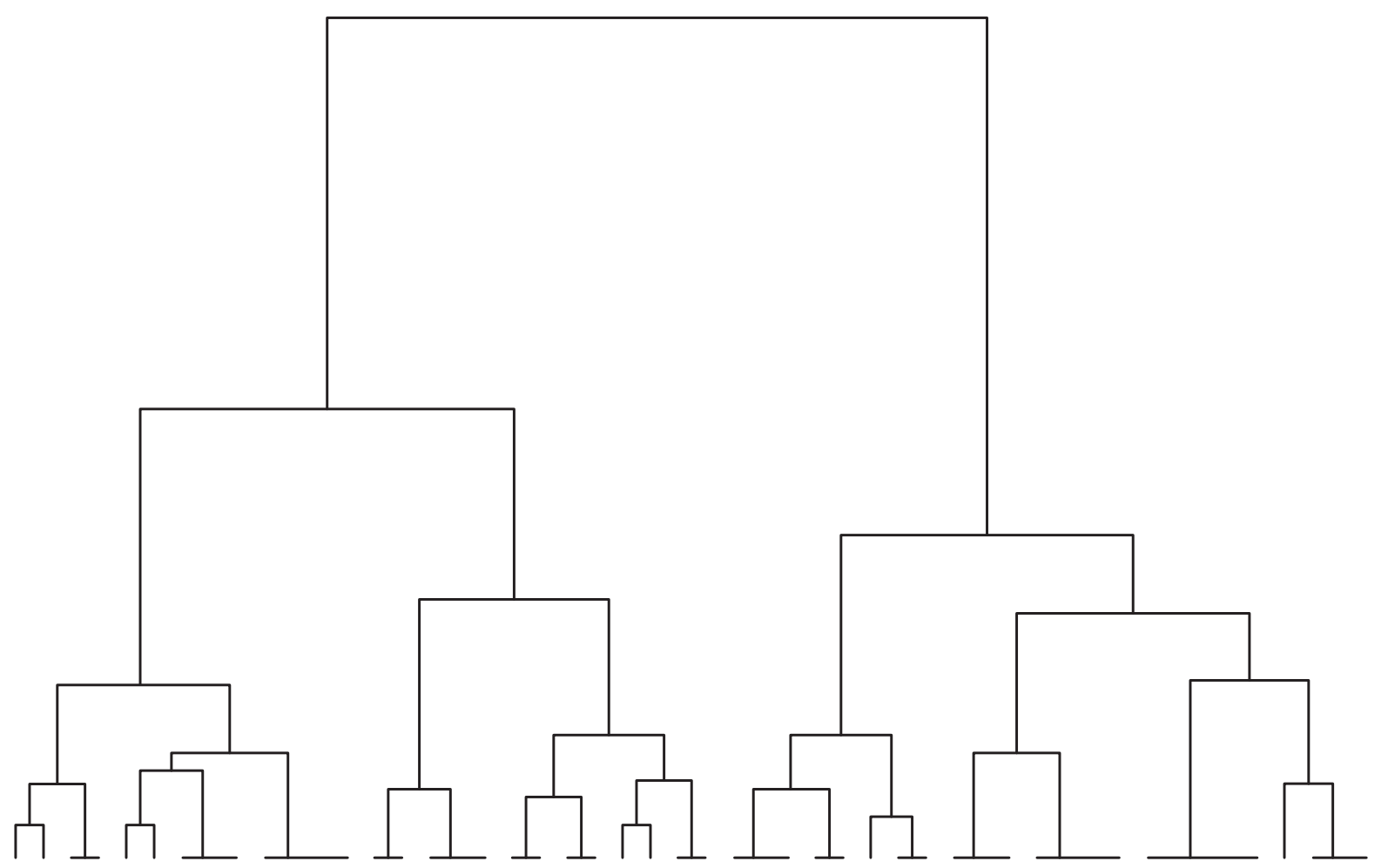

$124518313220101428276229161823151942124547112972136446343223554313302639413850371725 \quad 52483344$ Fuente: Elaboración propia (2017). 
Figura 3. Caracterización de conglomerados

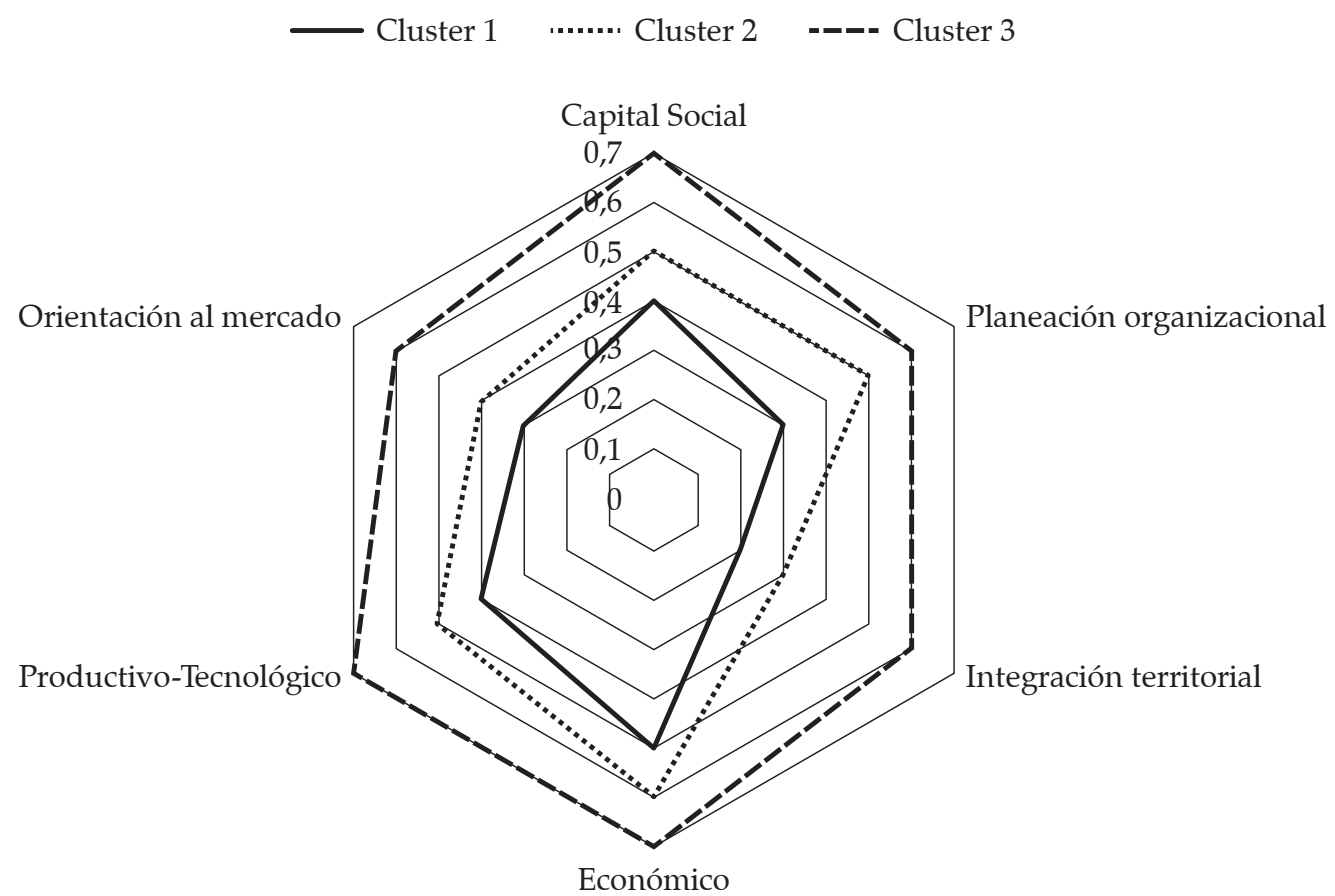

Fuente: Elaboración propia (2017).

\section{Discusión}

El análisis de la sostenibilidad de las agroempresas asociativas rurales a partir del índice de sostenibilidad Isoar, permitió identificar los procesos llevados a cabo en Nariño y Cauca como los de mayor sostenibilidad, sin encontrarse un patrón común en los dos casos en cuanto a los subcomponentes más destacados; en Nariño los más destacados fueron los subcomponentes integración territorial y económico, mientras en Cauca fueron Capital Social y Planeación organizacional.

Con relación a la integración territorial, en el caso de Nariño se destacan aspectos como la integración en red de 10 organizaciones de productores en una organización de segundo grado, lo cual permitió la implementación de un proceso de integración hacia atrás de la cadena productiva con suministro de insumos para la producción como semillas y agroquímicos por parte de la Federación hacia los asociados. Adicionalmente se promovieron espacios de intercambio de conocimientos y experiencias con otras organizaciones del departamento y de otros departamentos como Valle del Cauca y la tenencia de personal administrativo muy capacitado lo que les permitió tener gran capacidad de gestión para el acceso a recursos de convocatorias públicas y privadas de proyectos.

Estos resultados concuerdan con los hallazgos de otros autores en lo referente a la necesidad de consolidar redes de cooperación entre organizaciones de productores, buscando esquemas que permitan la generación de plataformas de servicios de apoyo técnico y financiero (IICA, 2009) y la acción colectiva para la compra de insumos, producción o comercialización (JOHNSON, SUAREZ y LUNDY, 2003) así como la implementación de mecanismos de intercambio de conocimientos y experiencias con otras organizaciones (LATTUADA, NOGUEIRA, RENOLD y URCOLA, 2011).

Desdeluego, la articulación de redes de cooperación genera externalidades positivas para las comunidades en torno al acceso a las fuentes de conocimiento (DE ARTECHE, SANTUCCI y WELSH, 2013), a partir de la configuración de estructuras que permiten promover el mejor desempeño económico de las organizaciones empresariales que trabajan de manera conjunta 
(MATTA, 2012). No obstante; en la integración territorial de las agroempresas a partir de sus redes, juega un rol destacado la fortaleza o debilidad de los lazos creados, donde más allá de las redes de proximidad, se pueden desarrollar capacidades interactivas que desbordan un ámbito local (BONO, 2009).

En cuanto al subcomponente económico, en el caso de Nariño se destacan aspectos comola implementación del plan de negocios para facilitar el acceso de la producción al mercado terminal, localizado a $472 \mathrm{~km}$ de distancia y la optimización de la logística para atender los canales de comercialización identificados antes de realizar la siembra; la implementación de un fondo rotatorio en especie para apoyar el suministro de insumos a los productores a través de un almacén de insumos agropecuarios; al igual que la generación de excedentes para el sostenimiento de la organización por la vía de comisión por venta de unidad de producto, cuotas de sostenimiento y las utilidades de la venta de los insumos. Adicionalmente la definición clara de la estructura de costos de la organización y el punto de equilibrio, acompañados de la definición de los márgenes de comercialización y un sistema de seguimiento y control a su cumplimiento.

Estos resultados concuerdan con los hallazgos de otros autores, en cuanto a la importancia del plan de negocios de la organización (AMÉZAGA et al., 2013) para generar excedentes que le permitan ofrecer servicios a sus asociados para favorecer la participación (BIALOSKORSKI, 2007); como asistencia técnica y proveeduría de insumos (ÁLVAREZ et al., 2012); mejoramiento de los ingresos de los productores por la optimización del canal de comercialización (IICA, 2009).

En el mismo sentido otros autores han reportado la importancia de la planificación estratégica de la organización (GOTTRET et al., 2011) para generar los recursos necesarios para su gestión empresarial por medio de cuotas de sostenimiento y comisión por venta del producto y el cumplimiento de los márgenes de comercialización así como la capacidad de gestión para el acceso a recursos de convocatorias públicas y privadas de proyectos, por lo cual son fundamentales las habilidades empresariales (ADRIAN y GREEN, 2001) y la experiencia administrativa del personal directivo de las organizaciones (AMOAH-MENSAH, 2013).

En el caso del Cauca, otra de las experiencias exitosas, se encontró como uno de los subcomponentes más destacados el capital social; en esta experiencia se implementaron estrategias participativas para involucrar a los productores en la identificación de sus necesidades y en el planteamiento de alternativas de solución a las mismas, se definieron reglas adecuadas al interior de la organización para evitar acciones oportunistas por parte de los asociados y mecanismos de intercambio de conocimientos y experiencias entre los asociados. Adicionalmente, se involucró a los productores de forma activa en los espacios administrativos y en la toma de decisiones, lo cual permitió la generación de relaciones de confianza.

El capital social definido como los vínculos generados entre actores, con soporte en la confianza y redes de relacionamientos (PUTNAM, 1993), permiten el alcance de ciertas metas, en cuya ausencia serían más difíciles de lograr, dado que el capital social es inherente a la estructura de las relaciones entre actores y dentro de grupos de actores (COLEMAN, 2000), pues la confianza y el capital social proveen el soporte de la cohesión, permitiendo relaciones de intercambio de diversos recursos en los agronegocios, así como ciertos tipos de transacciones (WILSON, 2000).

Los resultados en este subcomponente, concuerdan con los hallazgos de autores como Américo et al. (2013) quienes resaltan la pertinencia de utilizar metodologías participativas con los productores para la identificación de necesidades y el planteamiento de alternativas de solución para mejorar su compromiso con los procesos de intervención; Knack y Keefer (1997) plantean además, la importancia de las redes de contactos para el intercambio de conocimientos y experiencias, por la relación existente entre la calidad de las redes sociales y el bienestar económico.

Adicionalmente; la sostenibilidad de organizaciones asociativas rurales, también se puede ver influenciada por arreglos factibles de gobernanza y otros institucionales, que procuren por proteger los miembros asociados, través por ejemplo de mecanismos de resolución de conflictos, así como mecanismos de control que busquen la fidelidad de las personas que conforman la organización (ZYLBERSZTAJN, 2002).

Por su parte, Galvis y Rojas (2011) reportan además que cuando en las organizaciones se generan $\mathrm{y}$ consolidan estructuras sociales basadas en relaciones de confianza, en combinación de conductas de reciprocidad y cooperación, se fortalece el desarrollo de diversas actividades sociales, económicas y culturales; mientras Medici (2006) identificó como aspecto 
fundamental, el establecimiento de reglas adecuadas al interior de la organización para la ayuda mutua y el control social. Finalmente Durston (2000) plantea las normas y sanciones hacia individuos del colectivo, como mecanismos para la resolución de conflictos, gestión de recursos comunitarios y la generación de estructuras de trabajo en equipo.

Otro subcomponente destacado en el caso de Cauca, fue la planeación organizacional, en aspectos como la definición clara de una visión de futuro compartida y del objetivo de trabajo colectivo; la realización de reuniones periódicas para mantener informados a los productores y en especial la distribución de funciones entre los asociados por medio de comités y comisiones, involucrándolos a todos en el proceso de entrega el producto al mercado terminal para sensibilizarlos frente a la importancia de la calidad del producto y el manejo de poscosecha. Adicionalmente la tenencia de infraestructura para operación de la organización y la elaboración de planes como el plan estratégico y el plan operativo, al igual que la prestación de servicios a los productores, entre ellos la capacitación para el desarrollo de capacidades de liderazgo y capacidades para la acción colectiva.

Estos resultados concuerdan con lo reportado por la literatura como aspectos relevantes en la planeación para el fortalecimiento social; Bolaños (1999) plantea la importancia de analizar previamente las razones que tienen los productores para organizarse, sus sistemas de producción, mecanismos de comunicación, formas de organización y acción colectiva (CENTRO PARA EL DESARROLLO DE CAPITAL HUMANO, 2007) así como el grado de cohesión para la toma consensuada de decisiones (MORENO, URIBE y SANTIAGO, 2011).

De hecho; en esta discusión es preciso establecer que el rol de la planeación tiene también relación con el desarrollo de mecanismos internos de información dentro de la organización, con el fin de mejorar la toma de decisiones (ZYLBERSZTAJN, 1994).

En este mismo sentido, otros autores han reportado la importancia de la programación de reuniones periódicas para brindar información sobre la gestión de la organización favoreciendo la cooperación al interior del grupo y la participación activa de los beneficiarios en la toma de decisiones (DESAI y JOSHI, 2013); la distribución de funciones y la acción grupal para la toma de decisiones de manera colectiva (SZMULEWICZ, GUTIÉRREZ y WINKLER,
2012). Adicionalmente, la elaboración de instrumentos administrativos como el plan estratégico y el plan operativo (AMÉZAGA, RODRÍGUEZ, NÚÑEZ y HERRERA, 2013); la prestación de servicios como la información a los productores sobre el comportamiento de precios y mercados (RINCÓN et al., 2004).

Del mismo modo la literatura concuerda con los hallazgos de este estudio frente a la importancia para la consolidación de las organizaciones, del nivel educativo de los productores y el rol activo de los líderes (IICA, 2009); promoción del desarrollo de capacidades, liderazgo y acción colectiva (AMÉZAGA et al., 2013); y la realización de procesos de capacitación permanente como estrategia para lograr relaciones de familiaridad y confianza entre los asociados (ÁLVAREZ et al., 2012; Rincón et al., 2004) y la tenencia de infraestructura que facilite la operación de la organización (IICA, 2009).

Los dos subcomponentes menos destacados en todos los casos estudiados fueron el ProductivoTecnológico y la Orientación al mercado, en particular en Valle del Cauca y Antioquia. En lo referente al aspecto productivo-tecnológico, los aspectos que menos se tuvieron en cuenta en los procesos estudiados fueron la prestación de servicios de asistencia técnica para diversificar los ingresos y analizar los costos de producción; al igual que los aspectos ambientales como el análisis del impacto de las actividades realizadas por la organización y la implementación de planes de mitigación del impacto ambiental.

Estos resultados contrastan con los hallazgos de otros estudios que plantean la importancia de la asistencia técnica para satisfacer las demandas del mercado, disminuir el riesgo en la comercialización (IICA, 2009), analizar los costos de producción e implementar buenas prácticas agropecuarias; al igual que la implementación de procesos de acción colectiva para la producción (JOHNSON, SUAREZ y LUNDY, 2003), así como un enfoque de asesoramiento orientado al desarrollo de capacidades de autogestión (RODRÍGUEZ-ESPINOSA， RAMÍREZ-GÓMEZ y RESTREPO-BETANCUR, 2016).

En cuanto al otro subcomponente menos destacado en los procesos estudiados, la Orientación al mercado, los aspectos que menos se tuvieron en cuenta tienen relación con la planificación de la oferta de productos acordes con los requerimientos del mercado y el fortalecimiento de la capacidad financiera para pagar al productor en un tiempo razonable. 
Estos resultados concuerdan con los hallazgos de otros autores en cuanto a la necesidad de implementar estrategias que contribuyan a la sostenibilidad económica de la organización, como la planificación de la producción de acuerdo con los requerimientos del mercado en cuanto a calidad, volúmenes y continuidad de entregas (JOHNSON, SUAREZ y LUNDY, 2003).

Finalmente, los resultados de este estudio indican que el modelo menos sostenible de fortalecimiento de la organización agroempresarial rural fue el implementado en el Valle del Cauca, en el cual los subcomponentes de Capital Social y ProductivoTecnológico fueron los menos destacados.

El presente estudio por su enfoque cualitativo de carácter interpretativo, tiene como limitación que sus hallazgos no se pueden generalizar debido al tamaño de la muestra y la representatividad de los casos elegidos; no obstante, sus resultados como estudio preliminar sobre el fortalecimiento asociativo tienen validez como base para la implementación futura de investigaciones cuantitativas con muestreos probabilísticos que permitan la generalización de sus resultados por medio de la inferencia estadística.

\section{Conclusiones}

El enfoque multidimensional de sostenibilidad de las agroempresas asociativas rurales, pone en evidencia que las organizaciones más sostenibles en el tiempo han logrado fortalecer su capital social donde se afianzan relaciones de confianza y cooperación, logrando involucrar a sus productores asociados en esquemas de trabajo participativo y de intercambio. Por su parte ello contribuye a permitir la planeación organizacional, donde se generan mecanismos de organización productiva y social y la consolidación colectiva del plan estratégico y operativo de la organización empresarial, facilitando la toma de decisiones colectiva y consensuada. Aunque las organizaciones agroempresariales constituyen un actor más del territorio rural, su sostenibilidad en el tiempo tiene relación también con la integración territorial mediante la generación de redes de relaciones que permitan diversos procesos de intercambio con otros actores, así como economías de escala en los costos de producción. De tal manera que el componente económico ha jugado un rol importante en lo que respecta a aquellas organizaciones que logran diseñar un plan de negocios estableciendo la estructura de costo $\mathrm{y}$ beneficio, permitiendo reducir la incertidumbre y el riesgo de los asociados. Finalmente la consolidación de esas redes e interacciones tanto dentro de la organización como con otros actores ha permitido en aquellas organizaciones que son más sostenibles, alcanzar un mejoramiento productivo y tecnológico, una vez que acceden a servicios de capacitación, soporte y asistencia técnica, siempre que este fortalecimiento en lo productivo se pueda acompañar de una orientación al mercado mediante estrategias de comercialización. En todo caso la sostenibilidad de las agroempresas asociativas rurales se pueden soportar en un modelo multidimensional y no excluyente que constituyen pilares que pueden ser empleados en planes, programas o formulación de políticas públicas.

\section{Bibliografía}

ADRIAN, J. L. y GREEN, T. W. Agricultural Cooperative Managers and the Business Environment. Journal of Agribusiness, v. 1, spring 2001, p. 17-33, 2001.

ÁLVAREZ, Y. et al. La Cooperativa de Productores Agropecuarios de Lenguazaque, el Valle de Ubaté y municipios circunvecinos (Coopalac) y su impacto en el desarrollo rural del municipio de Lenguazaque, Cundinamarca. Gest. Soc., v. 5, n. 2, p. 51-73, 2012.

AMÉRICO, M. et al. A extensão rural na prática organizativa dos agricultores familiares da comunidade do Córrego do Mosquito, Jaguaré-ES. Cuadernos de Agroecología, v. 8, n. 2, p. 1-4, 2013.

AMÉZAGA, C. et al. Orientaciones estratégicas para el fortalecimiento de la gestión asociativa. San Salvador: IICA, 2013.

AMOAH-MENSAH, A. Strategic resources and performance of rural SMEs. International Journal of Business and Social Research, v. 3, n. 4, p. 106-119, 2013.

ARBER, S. Designing samples. In Researching social life. London: Sage, 1993, p. 68-93.

AYALA, J., ITURRALDE, T. y RODRÍGUEZ, A. Construcción de índices simplificados de riesgo país: el caso de Europa. Investigaciones Europeas de Dirección y Economía de la Empresa, v. 6, n. 1, p. 53-70, 2000.

BIALOSKORSKI, S. Um ensaio sobre desempenho econômico e participação em cooperativas agropecuárias. Revista de Economia E Sociologia Rural, 
v. 45 , n. 1 , p. $119-138$, 2007. http://doi.org/10.1590/ S0103-20032007000100006

BOLAÑOS, O. Caracterización y tipificación de organizaciones de productores y productoras. In XI Congreso Nacional Agronómico, 1999, p. 31-39.

BONO, J. La articulación local-global de sistemas territoriales de producción y de innovación. Revista de Estudios Regionales, n. 84, p. 53-82, 2009.

BORG, W. Educational research: an introduction. New York: Longman, 1989.

CENTRO PARA EL DESARROLLO DE CAPITAL HUMANO. Desarrollo de una metodología de fomento de la asociatividad del sector micro y pequeño empresarial, 2007.

COLEMAN, J. Social capital in the creation of human capital. In Social capital. A multifaceted perspective. Washington: World Bank, 2000.

DE ARTECHE, M., SANTUCCI, M. y WELSH, S. Redes y clusters para la innovación y la transferencia del conocimiento. Impacto en el crecimiento regional en Argentina. Estudios Gerenciales, v. 29, n. 127, p. 127-138, 2013. http://doi.org/10.1016/j.estger.2013.05.001

DENZIN, N. y LINCOLN, Y. Handbook of qualitative research. Handbook of Qualitative Research, v. 1, 1994.

DESAI, R. y JOSHI, S. (2013). Collective action and community development: evidence from self-help groups in rural India. The World Bank Economic Review, v. 28, n. 3, p. 492-524. http://doi.org/10.1093/wber/lht024

DURSTON, J. ¿Qué es el capital social comunitario? Serie Políticas Sociales, v. 38, n. 44, 2000.

EFENDIEV, A. y SOROKIN, P. Rural social organization and farmer cooperatives development in Russia and other emerging economies: comparative analysis. Developing Country Studies, v. 3, n. 14, p. 106-116, 2013.

ENSSLIN, S., ENSSLIN, L., IMLAU, J. y CHAVES, L. Processo de mapeamento das publicações científicas de um tema: portfólio bibliográfico e análise bibliométrica sobre avaliação de desempenho de cooperativas de produção agropecuária. Revista de Economia E Sociologia Rural, v. 52, n. 3, p. 587-608, 2014.

GALVIS, M. y ROJAS, J. Asociatividad, capital social y redes de innovación en la economía rural. Gestión Y Sociedad, v. 4, n. 1, p. 27-41, 2011.

GARZÓN, D., AMAYA, C. y CASTELLANOS, O. Modelo conceptual e instrumental de sostenibilidad organizacional a partir de la evaluación del tejido social empresarial. Innovar, v. 24, p. 82-93, 2004.

GOTTRET,M.,JUNKIN, R.y UGARTE, C. Autoevaluación facilitada para la gestión de empresas Asociativas rurales.
Turrialba, Costa Rica: Centro Agronómico Tropical de Investigación y Enseñanza, 2011.

IICA. Estudios de caso en comercialización de pequeños productores rurales. Bogotá: IICA-MADR, 2009.

KNACK, S. y KEEFER, P. Does social capital have an economic payoff? A cross-country investigation. The Quarterly Journal of Economics, p. 1251-1288, 1997.

LAMNEK, S. Qualitative social research. Berlin: Holz, 2005.

LATTUADA, M., NOGUEIRA, M., RENOLD, J. y URCOLA, M. El cooperativismo agropecuario argentino en la actualidad. Presentación y análisis de tres casos desde la perspectiva del capital social. Mundo Agrario, v. 12, n. 23, 2011.

LEMUS-AGUILAR, I. y HIDALGO, A. The design of a sustainable organization: a solid path through Innovation. In INGENIO Days 2016: Eu-SPRI Forum Early Career Researcher Conference (ECC): "Science, Innovation and the University: keys to social impact" 2016, p. 1-15.

MARRADI, A., ARCHENTI, N. y PIOVANI, J. Metodología de las ciencias sociales. Buenos Aires: Cengage Learning, 2007.

MARTÍNEZ-PELLITERO, M. y BAUMERT, T. Medida de la capacidad innovadora de las Comunidades Autónomas españolas: construcción de un índice regional de la innovación. Madrid, 2003. Retrieved from: <http:// www.ucm.es/bucm/cee/iaif>.

MATTA, A. Aportes del análisis de redes sociales a la gestión de estrategias de cooperación empresarial. REDES - Revista Hispana Para El Análisis de Redes Sociales, v. 23, p. 146-177, 2012. Retrieved from: < http:// revistes.uab.cat/redes/article/view/442/332>.

MEDICI, A. Empreendedorismo e associativismo nos agronegócios: a sua presença (e ausência) na região de Botucatu-SP. Revista Ciência em Extensão, v. 3, n. 1, p. 70-80, 2006.

MELGAREJO, Z., VERA-COLINA, M. y MORARIAPIRA, E. Competitividad de la MIPYME y desarrollo regional. Estudio del caso colombiano. Tendencias, v. XIV, n. 2, p. 184-215, 2013.

MINISTERIO DE AGRICULTURA Y DESARROLLO RURAL. Índice de Capacidad Organizacional - ICO. Bogotá: Proyecto piloto-Zonas de Reserva Campesina, 2003.

MORENO,D.,URIBE, M.y SANTIAGO, L. Comunicación y manejo social para la asociatividad: mecanismo para la sostenibilidad rural. Bogotá: SENA-SAC, 2011. 
MUÑOZ, M., SANTOYO, V. y FLORES, J. Pilares de las organizaciones rurales que perduran, Vol. 90, 2010.

MURCIA, H. Agribusiness model approach to territorial food development. Agronomía Colombiana, v. 29, n. 1, p. 125-132, 2011.

NOELLE-NEUMANN, E. y PETERSEN, T. Alle, nicht jeder. Einführung in die Methoden der Demoskopie. 4. ed. Berlin: Springer-Verlag, 2005.

OECD. Innovation and Modernising the Rural Economy. OECD Publishing, 2014. http://doi.org/http:// dx.doi.org/10.1787/9789264205390-en

OSTERTAG, C. Factores claves de éxito de una agroindustria rural: el caso de la asociación de producción y mercadeo para la educación - Asprome. Santiago de Chile: FAO, 2002.

PUTNAM, R. Making Democracy work. Civic traditions in Modern Italy. Princeton: Princeton University Press, 1993.

QUANG, T. y ITAGAKI, K. Agro-enterprise performance and rural investment climate: evidence from the north of Vietnam. International Business and Management, v. 5, n. 2, p. 28-36, 2012. http://doi. org/10.3968/j.ibm.1923842820120502.1055

RIELLA, A. y VITELLI, R. Organizaciones rurales y acción colectiva en Uruguay: estudios en tiempos de crisis (1a). Montevideo: Europe Aid Co-operation Office, 2009.

RINCÓN, N. et al. (2004). Los pequeños productores y su participación en el proceso de comercialización agrícola. Revista de La Facultad de Agronomía, v. 21, n. 2, p. 1-13.

RODRÍGUEZ-ESPINOSA, H., RAMÍREZ-GÓMEZ, C. y RESTREPO-BETANCUR, L. Nuevas tendencias de la extensión rural para el desarrollo de capacidades de autogestión. Corpoica Ciencia y Tecnología Agropecuaria, v 17, n. 1, p. 31-42, 2016. http://doi.org/10.21930/rcta. vol17_num1_art:457

RODRÍGUEZ, H., RAMÍREZ, C. y RESTREPOBETANCUR, L. Propuesta metodológica para la formulación participativa de programas de desarrollo agropecuario local. Revista Luna Azul, v. 40, n. 1, p. 134164, 2015. http://doi.org/10.17151/luaz.2015.40.15
RODRÍGUEZ, H. y RAMÍREZ, C. Análisis de la sostenibilidad de los procesos de fortalecimiento de la asociatividad rural: el caso de Asomora. Revista de Ciencias Agrícolas, v. 33, n. 1, p. 10-22, 2016.

RUEDA, M. y MUÑOZ, J. Asociatividad, capital social y redes de innovación en la economía rural. Gestión Social, v. 4, n. 1, p. 27-41, 2011.

RUGELES, L. y JOLLY, J. Hacia la construcción de modelos agroempresariales en una perspectiva territorial. Cuadernos de Administración, v. 19, n. 32, p. 295-317, 2006. Retrieved from: <http://revistas. javeriana.edu.co/index.php/cuadernos_admon/article/ download/4316/3270>.

SOCIEDAD DE AGRICULTORES DE COLOMBIA. Sector rural y agropecuario: desarrollo y futuro. Bogotá, 2010.

SOLARTE, G. Asociatividad, empresarización y pactos territoriales: claves del desarrollo de los territorios rurales. Corporación Lationamericana Misión Rural, 2011.

SPIELMAN, D. y KELEMEWORK, D. Measuring agricultural innovation system properties and performance: Illustrations from Ethiopia and Vietnam. IFPRI Discussion Paper 851. Washington, D.C., 2009.

SZMULEWICZ, P., GUTIÉRREZ, C. y WINKLER, K. Evaluación de las habilidades asociativas en redes de Agroturismo del sur de Chile. Estudios y Perspectivas en Turismo, v. 21, p. 1013-1034, 2012.

USAID. Una herramienta de diagnóstico para el fortalecimiento de organizaciones de productores. Lima, Perú, n/d.

WILSON, P. Social capital, trust, and the agribusiness of economics. Journal of Agricultural and Resource Economics, v. 25, n. 1, p. 1-13, 2000.

YIN, R. Case study research: design and methods. 5. ed. Los Angeles: Sage publications, 2013.

ZYLBERSZTAJN, D. Organização de cooperativas: desafios. Revista de Administração, v. 29, n. 3, p. 23-32, 1994.

- Quatro estratégias fundamentais para cooperativas agrícolas. Agronegócio cooperativo -reestruturação e estratégias. Viçosa, MG: UFV, 2002.

Todo o conteúdo deste periódico, exceto onde estiver identificado, está licenciado sob uma Licença Creative Commons (cc by 4.0$)$ 
